\title{
Meta-analysis of mRNA expression profiles to identify differentially expressed genes in lung adenocarcinoma tissue from smokers and non-smokers
}

\author{
XIAONA HE $^{1 *}$, CHENG ZHANG ${ }^{2 *}, \mathrm{CHAO} \mathrm{SHI}^{2}$ and QUQIN LU ${ }^{1}$ \\ ${ }^{1}$ Department of Biostatistics and Epidemiology, School of Public Health, Nanchang University; \\ ${ }^{2}$ Center for Experimental Medicine, The First Affiliated Hospital of Nanchang University, \\ Nanchang, Jiangxi 330006, P.R. China
}

Received June 26, 2017; Accepted December 29, 2017

DOI: $10.3892 /$ or.2018.6197

\begin{abstract}
Compared to other types of lung cancer, lung adenocarcinoma patients with a history of smoking have a poor prognosis during the treatment of lung cancer. How lung adenocarcinoma-related genes are differentially expressed between smoker and non-smoker patients has yet to be fully elucidated. We performed a meta-analysis of four publicly available microarray datasets related to lung adenocarcinoma tissue in patients with a history of smoking using $\mathrm{R}$ statistical software. The top 50 differentially expressed genes (DEGs) in smoking vs. non-smoking patients are shown using heat maps. Additionally, we conducted KEGG and GO analyses. In addition, we performed a PPI network analysis for 8 genes that were selected during a previous analysis. We identified a total of 2,932 DEGs (1,806 upregulated, 1,126 downregulated) and five genes (CDC45, CDC20, ANAPC7, CDC6, ESPL1) that may link lung adenocarcinoma to smoking history. Our study may provide new insights into the complex mechanisms of lung adenocarcinoma in smoking patients, and our novel gene expression signatures will be useful for future clinical studies.
\end{abstract}

\section{Introduction}

Lung cancer is one of the most common types of cancer and is the leading cause of cancer-related mortality wordwide. Small cell lung cancer (SCLC) and non-small cell lung cancer (NSCLC) are the most common types of lung cancer, of which NSCLC accounts for approximately $85 \%$ of all

Correspondence to: Dr Quqin Lu, Department of Biostatistics and Epidemiology, School of Public Health, Nanchang University, 460 Bayi Avenue, Donghu, Nanchang, Jiangxi 330006, P.R. China E-mail: quqinlu@ncu.edu.cn

*Contributed equally

Key words: meta-analysis, microarray, differentially expressed genes, lung adenocarcinoma, smoking cases (1). Lung adenocarcinoma is the most common subtype of NSCLC $(40 \%)$ in many countries $(2,3)$. To date, many genetic factors have been proposed to be involved in lung adenocarcinoma, including several tumour-suppressor genes (TP53, CDKN2A, STK11, NF1, ATM, RB1, and APC) $(4,5)$. Several new targeted therapies have resulted in considerable clinical benefits for cancer patients in recent years, as well as a deeper understanding of lung adenocarcinoma at the molecular level. One example of a new targeted therapy is epidermal growth factor receptor (EGFR) and KRAS targeted gene therapy $(6,7)$. However, targeted gene therapy is mainly used when patients have special characteristics. EGFR mutations occur more frequently in female lung adenocarcinoma patients with a non-smoking history (8). HER 2 mutations tend to occur in non-smoking males (9). In contrast, KRAS mutations occur during the early development of smoking-related lung adenocarcinoma (10). Based on these observations, there is a need to develop individualized treatment programs for patients with unique clinical characteristics. Lung adenocarcinoma is caused by a combination of genetic and environmental effects (11).

More recently, the incidence of lung adenocarcinoma has increased in smokers (12). Tobacco smoke contains a mixture of harmful compounds and carcinogens (13). Therefore, smoking plays an important role in the development of lung adenocarcinoma. Although the correlation between smoking and lung adenocarcinoma has been demonstrated in previous studies, a meta-analysis of the gene mutations in a large number of tissue samples that considers the smoking history in lung adenocarcinoma has not yet been conducted (14). This large scale analysis can reduce the differences caused by different research conditions and can integrate the results from previous studies to evaluate the issue from another point of view. The development of microarray methods for large scale analysis of gene expression makes it possible to perform a more comprehensive analysis for potential genes and molecular pathways associated with lung adenocarcinoma in smoking patients (15). DNA microarray analysis has been applied to investigate whole genomic expression profiles and physiological mechanisms in health and disease $(16,17)$. Therefore, a high-throughput microarray experiment was designed to 
analyse the genetic expression patterns and identify potential genes to target for lung adenocarcinoma (18). Meta-analysis provides a powerful tool for analysing microarray experiments by combining data from multiple studies (19). Genes identified by meta-analysis tend to overlap with genes identified in other studies, suggesting increased reliability (20). In addition to providing a new perspective, this research topic will further the understanding of the relationship between smoking and lung adenocarcinoma.

The aim of this study was to identify possible candidate genes for personalized treatment for lung adenocarcinoma patients with a history of smoking to provide patients with better treatment options and ensure a good prognosis. Therefore, we conducted a meta-analysis using the same platform of gene expression profile data that associated smoking with lung adenocarcinoma tissue.

\section{Materials and methods}

Selection of microarray datasets for meta-analysis. According to the Preferred Reporting Items for Systematic Reviews and Meta-Analysis (PRISMA) guidelines published in 2009, we performed a detailed and comprehensive search of microarray datasets in the Gene Expression Omnibus (GEO) database of the National Center for Biotechnology Information (NCBI) (http://www.ncbi.nlm.nih.gov/geo/).

Meta-analysis data. To maintain objectivity, the data were simultaneously extracted by two independent reviewers from the original search. Any discrepancies that arose between the two reviewers were resolved by consultation with a third reviewer. The terms 'lung neoplasms' and 'lung cancer' were considered keywords during our search for this study. In addition, studies that reported non-human data were excluded in the selection process for microarray datasets. Finally, 583 datasets were obtained from searching the Gene Expression Omnibus (GEO) database. Datasets with $>20,288$ samples were elected for the study. We included a dataset in the meta-analysis if it contained i) all samples on the Affymetrix Human Genome U133 Plus 2.0 Array platform, ii) samples from lung adenocarcinoma tissue and iii) samples with valid smoking statuses. According to the criteria, the four datasets that were selected from the 288 datasets included 477 lung adenocarcinoma tissues with valid smoking statuses. Then, we downloaded the lung adenocarcinoma tissue files (CEL) of the four microarray datasets from the GEO database with accession numbers GSE12667, GSE31210, GSE40791, and GSE50081. The four datasets included 477 lung adenocarcinoma patients; 327 of which were smokers, and 150 were non-smokers; the smokers included former smokers, current smokers and ex-smokers.

Meta-analysis of microarray datasets using the same platform . We conducted the meta-analysis of gene expression profiles of the selected four microarray datasets by using R statistical software (http://www.r-project.org/) with the same platform. Prior to the meta-analysis, we performed data normalization of the four datasets using R statistical software. Then, we processed the meta-analysis using the MAMA, mataMA, affyPLM and CLL packages in $\mathrm{R}$ statistical software according to the $\mathrm{t}$-test

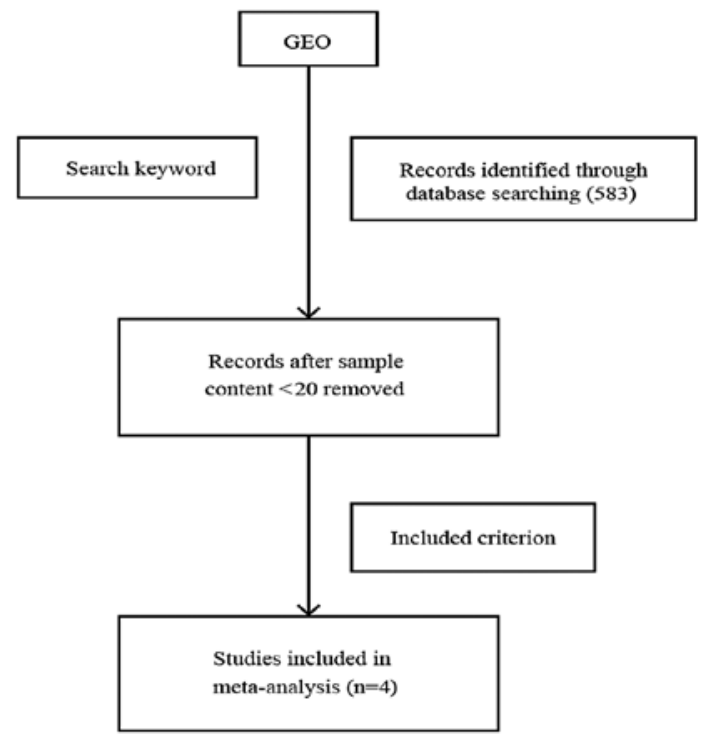

Figure 1. Selection process of the microarray datasets for meta-analysis of lung adenocarcinoma tissue with smoking status.

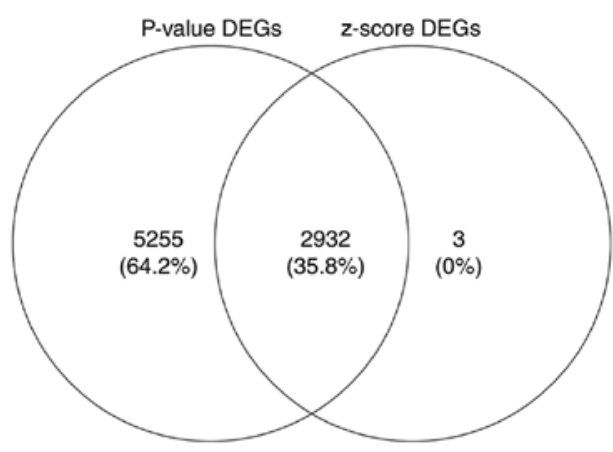

Figure 2. The 2932 overlapping differentially expressed genes (DEGs) based on P-value (where the threshold was $<0.005$ ) and z-score (where the threshold was an absolute value $>3$ ) were detected using Venny 2.1.0.

and z-score methods. During the meta-analysis with R statistical software, a list of differentially expressed genes (DEGs) (upregulated or downregulated) were identified based on the $\mathrm{P}$-values (where the threshold was $<0.005$ ) and $\mathrm{z}$-scores (where the threshold was an absolute value $>3$ ).

Enrichment analysis of the GO function and KEGG pathway. It is important to understand the biological implications of the identified DEGs in lung adenocarcinoma tissue. According to the meta-analysis results, the most significant 200 DEGs (100 upregulated and 100 downregulated) were selected for enrichment analysis. Then, we conducted the functional enrichment analysis of the gene ontology (GO) function and the Kyoto Encyclopedia of Genes and Genomes (KEGG) pathway using the WEB-based GEne SeT AnaLysis Toolkit (http://bioinfo.vanderbilt.edu/webgestalt/login.php) under a significance threshold of $\mathrm{P}<0.05$.

PPI network analysis. To further understand and predict the biological activity of the identified DEGs that were based on the results of the GO function and KEGG pathway enrichment analyses, we conducted a protein-protein interaction (PPI) 
Table I. Characteristic of individual studies retrieved from Gene Expression Omnibus for meta-analysis.

\begin{tabular}{|c|c|c|c|c|}
\hline \multirow[b]{2}{*}{ Dataset } & \multicolumn{2}{|c|}{ Sample } & \multirow[b]{2}{*}{ Tissue } & \multirow[b]{2}{*}{ Platform } \\
\hline & Smoking status & Non-smoking status & & \\
\hline GSE12667 & 40 & 8 & Lung adenocarcinoma & Affymetrix Human Genome U133 Plus 2.0 Array \\
\hline GSE31210 & 111 & 115 & Lung adenocarcinoma & Affymetrix Human Genome U133 Plus 2.0 Array \\
\hline GSE40791 & 82 & 4 & Lung adenocarcinoma & Affymetrix Human Genome U133 Plus 2.0 Array \\
\hline GSE50081 & 94 & 23 & Lung adenocarcinoma & Affymetrix Human Genome U133 Plus 2.0 Array \\
\hline
\end{tabular}

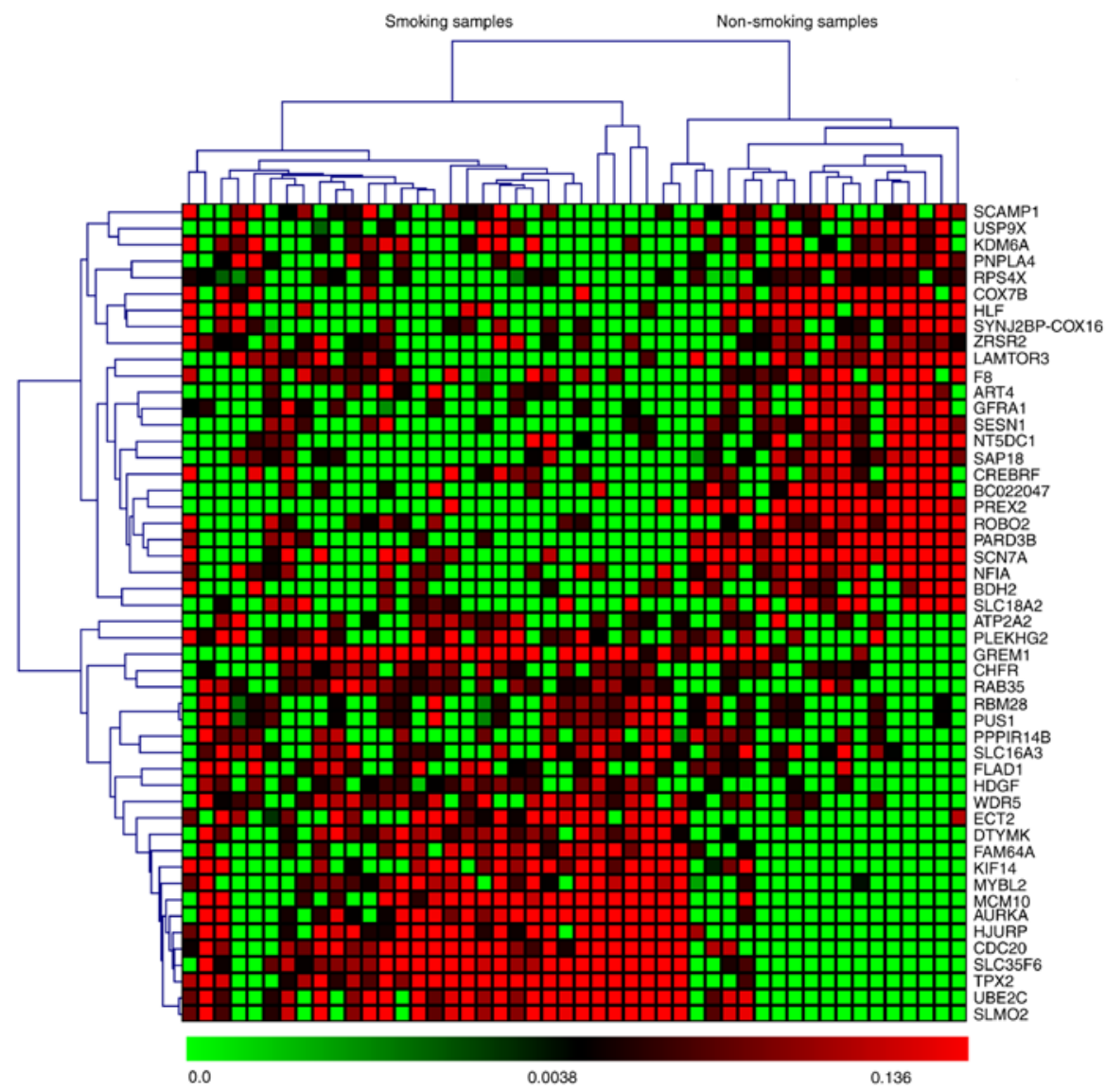

Figure 3. Heat-map representation of the expression profiles for the top 25 upregulated and downregulated genes in the GSE12667 dataset. The clustering of the selected genes on the heat-map was performed by using a hierarchical clustering algorithm that uses an average linkage method and Pearson's correlation coefficient.

network using the Cytoscape software. Prior to this analysis, we imported the DEG-encoding proteins into a protein-protein interaction (PPI) network, which was downloaded from the Biological General Repository for Interaction Datasets (BioGRID, http://thebiogrid.org/).

\section{Results}

Selection of microarray datasets related to lung adenocarcinoma for meta-analysis. From the microarray datasets retrieved from the GEO database of NCBI, we extracted 477 GEO lung adenocarcinoma samples that belonged to four microarray datasets, which met our criteria for metaanalysis (see Materials and methods, and Fig. 1). All four GEO series (GSEs) were microarray datasets that used only lung adenocarcinoma tissue with valid smoking statuses. The GEO Platform Files (GPLs) from the four datasets (GSE12667, GSE31210, GSE40791 and GSE50081) were obtained using the Affymetrix 'Gene Chip' (Table I).

Identification of upregulated or downregulated DEGs through meta-analysis. We performed the meta-analysis of gene expression profiles according to t-test and z-score methods using MAMA, mataMA, affyPLM and CLL packages in $\mathrm{R}$ statistical software on the same platform. According to the P-value (where the threshold was $<0.005$ ) and z-score (where the threshold was an absolute value $>3$ ), we were able to identify a total of 2,932 DEGs, including 1,806 upregulated and 1,126 downregulated genes using Venny 2.0 (http:// bioinfogp.cnb.csic.es/tools/venny/index.html). The 200 genes 


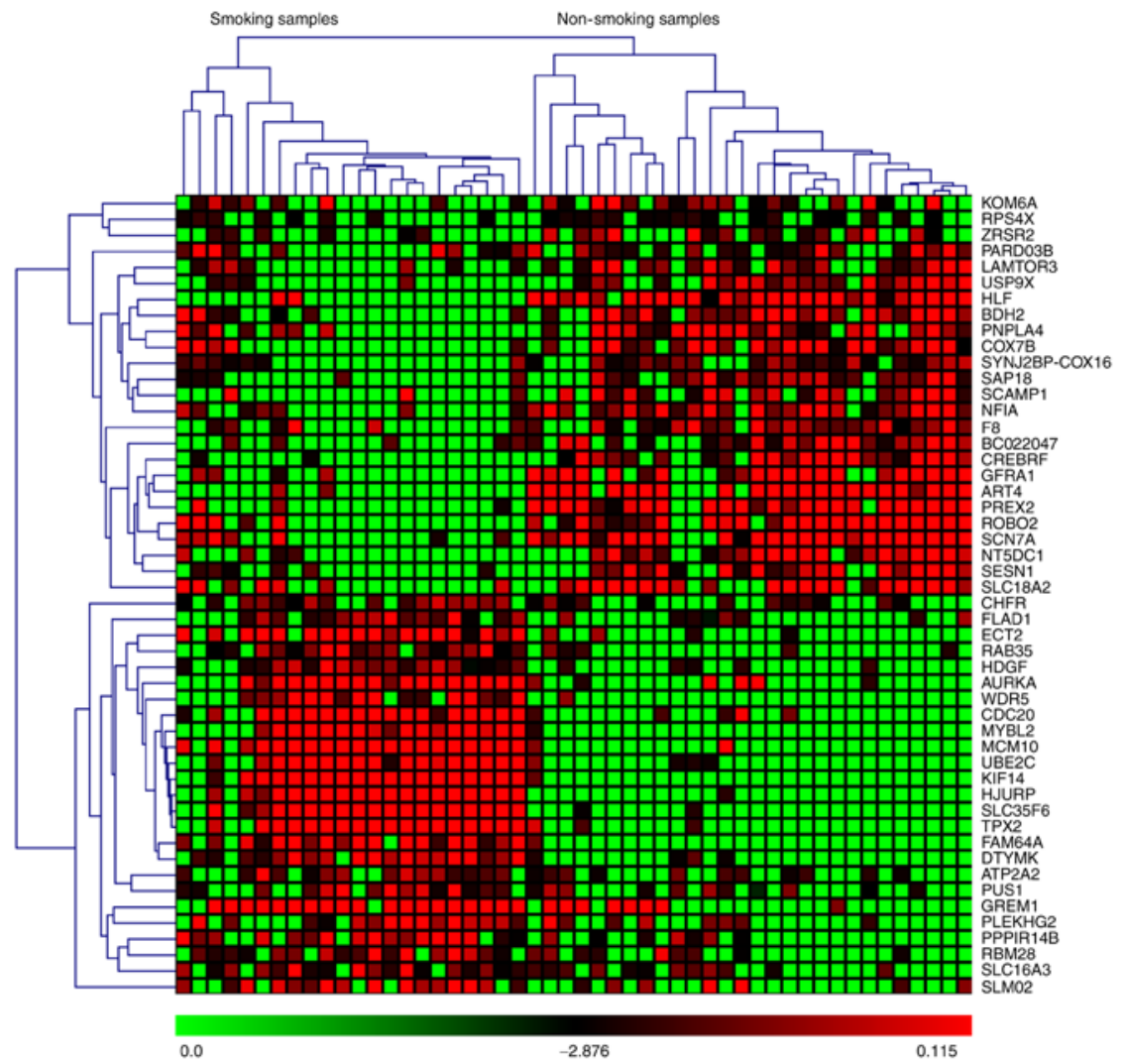

Figure 4. Heat-map representation of the expression profiles for the top 25 upregulated and downregulated differentially expressed genes (DEGs) in the GSE131210 dataset. The clustering of the selected genes on the heat-map was performed using a hierarchical clustering algorithm that uses an average linkage method and Pearson's correlation coefficient.

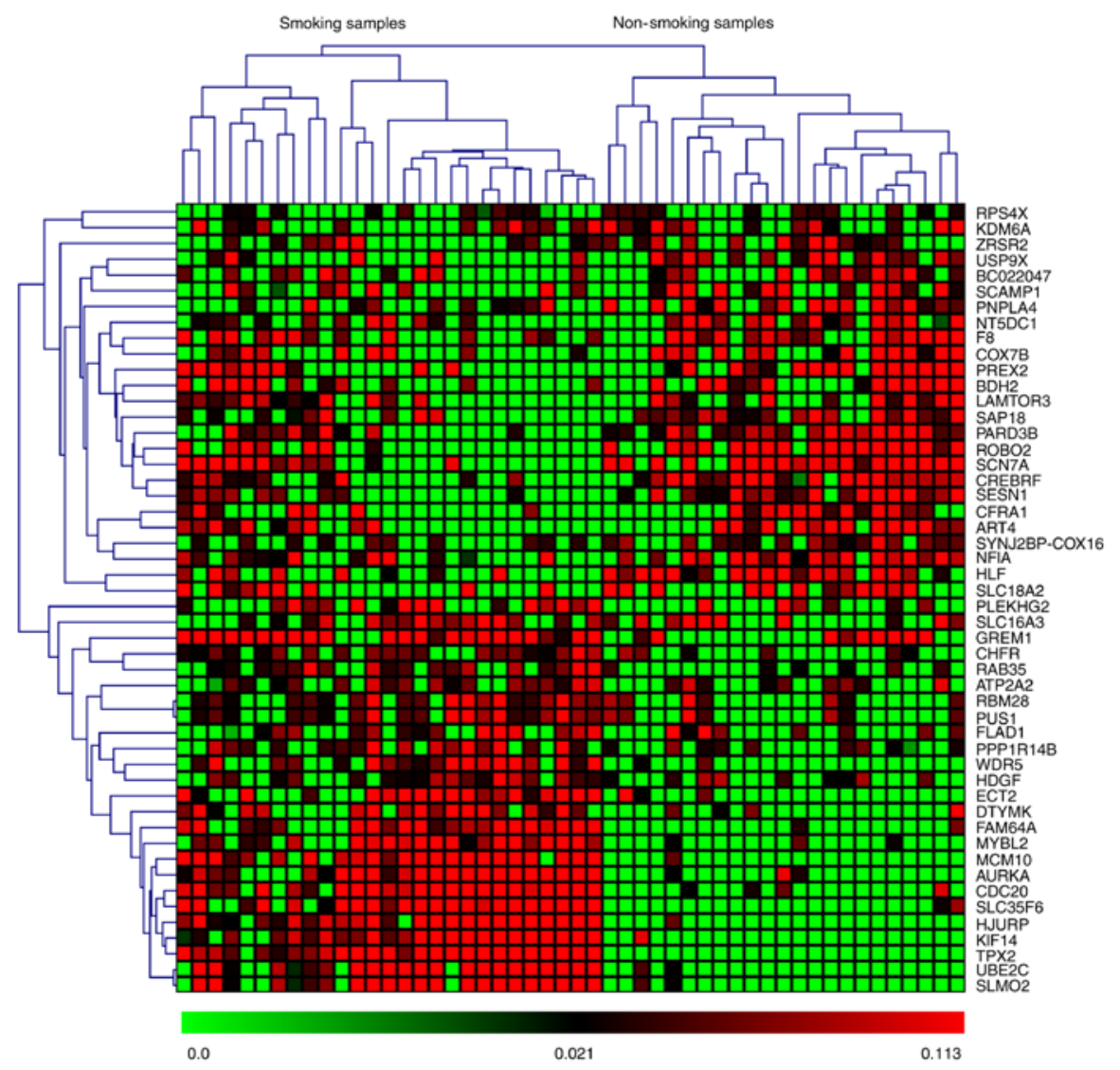

Figure 5. Heat-map representation of the expression profiles for the top 25 upregulated and downregulated differentially expressed genes (DEGs) in the GSE40791 dataset. The clustering of the selected genes on the heat-map was performed using a hierarchical clustering algorithm that uses an average linkage method and Pearson's correlation coefficient. 


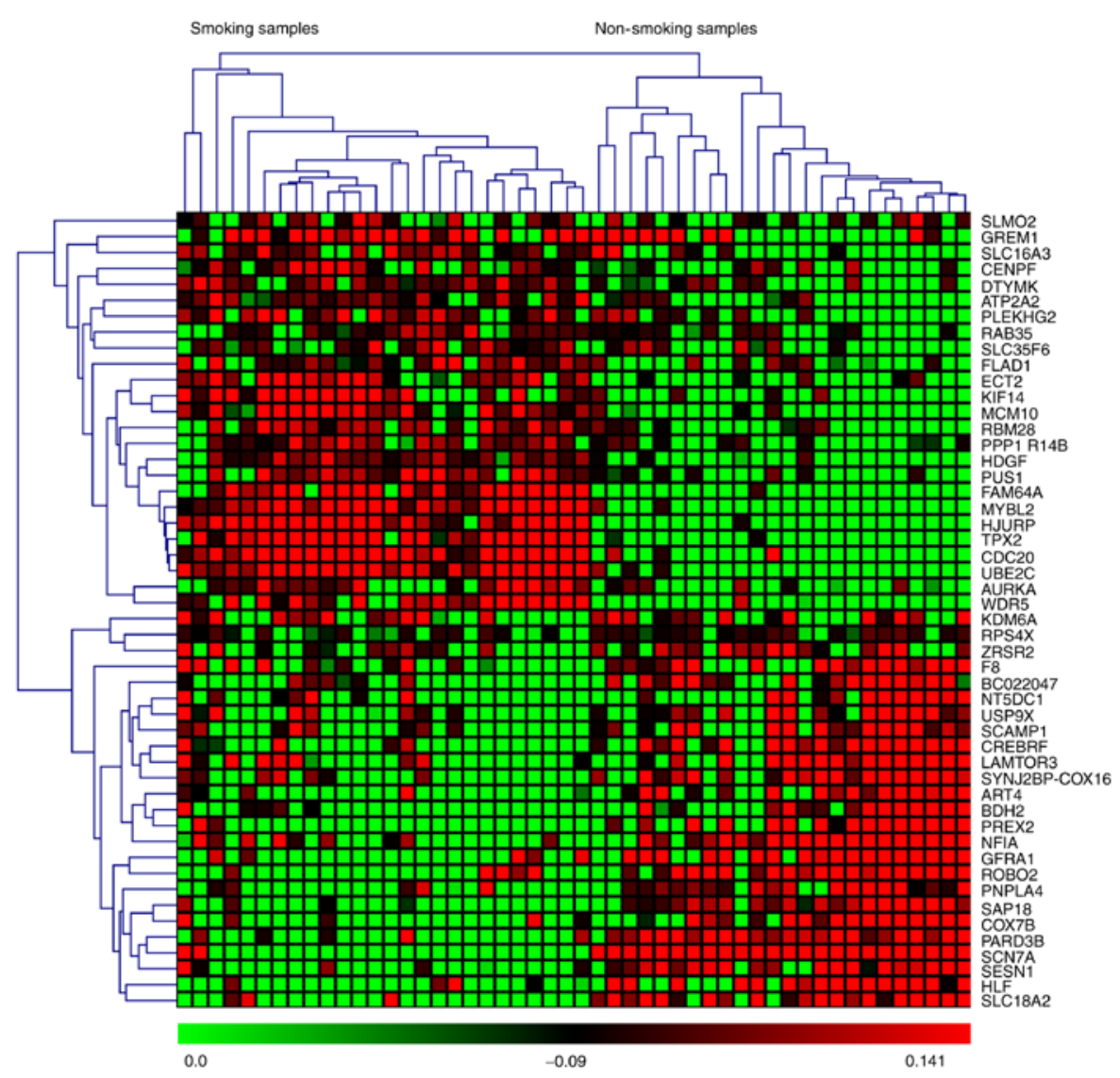

Figure 6. Heat-map representation of the expression profiles for the top 25 upregulated and downregulated differentially expressed genes (DEGs) in the GSE50081 dataset. The clustering of the selected genes on the heat-map was performed using a hierarchical clustering algorithm that uses an average linkage method and Pearson's correlation coefficient.

that showed maximum upregulation and downregulation are shown in Tables II and III, and the overlapping DEGs based on P-values and z-scores are shown in Fig. 2. A subset of the top 50 DEGs (25 upregulated and 25 downregulated) in the four microarray datasets were visualized with heat maps using the Mev software and are shown in Figs. 3-6.

Enrichment analysis of the GO function and KEGG pathway for the top 100 upregulated and downregulated DEGs. We classified the 200 DEGs that were identified through meta-analysis according to the GO hierarchy into functional categories (biological process, molecular function, and cellular component) and based on the KEGG pathway, with a significance threshold of $<0.05$. The most significant GO terms under the biological processes category were enriched in the following descending order: 'cell cycle phase' (GO:0022403), 'M phase of mitotic cell cycle' (GO:0000087) and 'mitotic cell cycle' (GO:0000278). The most enriched GO terms under the molecular functions and cellular components categories were 'protein binding' (GO:0005515) and 'nuclear part' (GO:0044428). The most enriched KEGG pathway terms were (in descending order): 'Cell cycle' (kegg:04110), 'Oocyte meiosis' (kegg:04114) and 'Ubiquitin mediated proteolysis' (kegg:04120) (Tables IV and V).

PPI network analysis of the DEGs. To understand the biological meaning of the 8 upregulated DEGs identified by the KEGG pathway under the cell cycle pathway at the protein level, we constructed a PPI network for the proteins encoded by the 8 DEGs with interactions that included 541 nodes and 671 edges as shown in Fig. 7.

\section{Discussion}

In the present study, we showed that genes are differentially expressed in lung adenocarcinoma in smoking and non-smoking patients. Some genes that showed the highest expression levels were found in lung adenocarcinoma patients who had a smoking history. Smoking consistently plays an important role in the development of lung adenocarcinoma. Cigarette smoke contains over 400 identified chemicals, at least 250 of which are implicated in tumour initiation and promotion (21). It is estimated that more than 50 chemicals in tobacco smoke cause cancers (22). Cigarette smoke is by far the most widespread link between exposure to known carcinogens and death from lung cancer (23). Lung adenocarcinoma is one of the main types of lung cancer in smokers and cannot be successfully treated with traditional treatments. Therefore, the effects of cigarette smoke on the genes that are implicated in lung adenocarcinoma are critical to increase our understanding of the carcinogenesis and in finding targeted genes. In our study, we found that the cell cycle pathway was significantly altered in lung adenocarcinoma tissues from patients with a smoking history. 
Table II. The 100 upregulated genes.

\begin{tabular}{|c|c|c|c|c|c|c|c|}
\hline Probe ID & Gene & P-value & Z-score & Probe ID & Gene & P-value & Z-score \\
\hline 218670_at & PUS1 & $1.26565 \mathrm{E}-14$ & -3.364765896 & 204603_at & EXO1 & $2.21381 \mathrm{E}-11$ & -3.0 \\
\hline 202856_s_at & SLC16A3 & $1.31006 \mathrm{E}-14$ & -3.005755138 & 225401_at & C1orf85 & $2.37168 \mathrm{E}-11$ & -4.583223012 \\
\hline 1553984_s_at & DTYMK & $2.73115 \mathrm{E}-14$ & -3.77721059 & 228703_at & P4HA3 & $2.44789 \mathrm{E}-11$ & -4.354770166 \\
\hline 210052_s_at & TPX2 & $3.28626 \mathrm{E}-14$ & -3.156028484 & 204709_s_at & KIF23 & $2.78617 \mathrm{E}-11$ & -3.130038648 \\
\hline 225620_at & RAB35 & $6.72795 \mathrm{E}-14$ & -3.977400883 & 212322_at & SGPL1 & $3.15128 \mathrm{E}-11$ & 29755 \\
\hline 201710_at & MYBL2 & $1.13465 \mathrm{E}-13$ & -3.753904206 & 202779_s_at & UBE2S & $3.25431 \mathrm{E}-11$ & -3.2 \\
\hline 200896_x_at & HDGF & $1.32117 \mathrm{E}-13$ & -6.606272774 & 210386_s_at & MTX1 & 3.28 & -3. \\
\hline 233986_s_at & PLEKHG2 & $1.34559 \mathrm{E}-13$ & -4.721664344 & 205733_at & & $3.44063 \mathrm{E}-11$ & 7987 \\
\hline 209186_at & ATP2A2 & $1.52767 \mathrm{E}-13$ & -3.331133151 & 223307_at & $\mathrm{CDCA}$ & 3.49 & 1207 \\
\hline 202954_at & UBE2C & $1.96732 \mathrm{E}-13$ & -3.43 & 43 at & $\mathrm{PG}$ & 3.49 & -4 \\
\hline 234992_x_at & ECT2 & $2.22933 \mathrm{E}-13$ & -3.540186 & 3_at & $\mathrm{P} 1$ & 3. & 777 \\
\hline 218468_s_at & GREM & $2.91323 \mathrm{E}-13$ & -3.4 & 223785_at & & $4.13012 \mathrm{E}-11$ & -3.7 \\
\hline 221591_s_at & FAM64A & $3.1064 \mathrm{E}-13$ & -3.64 & 212021_s_at & MKI67 & $4.16123 \mathrm{E}-11$ & -3.291213712 \\
\hline 223308_s_at & WDR5 & $3.71925 \mathrm{E}-13$ & -3.44 & 200750_s_at & RAN & $4.22222 \mathrm{E}-11$ & -3.060882727 \\
\hline 204092_s_at & AURKA & $4.20552 \mathrm{E}-13$ & 115008 & 229892_at & EP400NL & 4.39129E-11 & -4.569469931 \\
\hline 218593_at & RBM28 & $5.6688 \mathrm{E}-13$ & -3.725504934 & 204126_s_at & CDC45 & $1 \mathrm{E}-11$ & 729352 \\
\hline 204962_s_at & SLC35F6 & $6.05294 \mathrm{E}-13$ & -3.16224673 & 226949_at & GOLGA3 & & -3.5 \\
\hline 218726_at & HJURP & $9.13047 \mathrm{E}-13$ & & 205895_s_at & NOL & $3 \mathrm{E}-11$ & 9055682 \\
\hline 206364_at & KIF14 & $1.22724 \mathrm{E}-12$ & & 205691_at & SYNG & -11 & 1404 \\
\hline 202870_s_at & CDC20 & $1.31761 \mathrm{E}-12$ & & 2046 & $\mathrm{NEK}$ & -11 & 411 \\
\hline 212680_x_at & PPP1R14B & $1.41753 \mathrm{E}-12$ & -3.30 & 223365_at & DHX37 & $5.08806 \mathrm{E}-11$ & 2983 \\
\hline 220651_s_at & MCM10 & $1.66711 \mathrm{E}-12$ & -3.962832885 & 229610_at & $\mathrm{CK}$ & $1 E-11$ & 0101 \\
\hline 222441_x_at & SLMO2 & $1.88827 \mathrm{E}-12$ & -3.580783528 & 207590_s_at & CEN & $1 \mathrm{E}-11$ & 8048 \\
\hline 212541_at & FLAD1 & $2.68452 \mathrm{E}-12$ & -4.335857984 & 42_at & ABHD12 & $6.35478 \mathrm{E}-11$ & -3.351775356 \\
\hline 223931_s_at & CHFR & $2.91989 \mathrm{E}-12$ & -5.133 & _S_at & WH & $6.63429 \mathrm{E}-11$ & 5902 \\
\hline 203612_at & BYSL & $2.94276 \mathrm{E}-12$ & -3.3325 & -_S_at & HMC & $6.86768 \mathrm{E}-11$ & 687751 \\
\hline 219874_at & SLC12A8 & $3.14992 \mathrm{E}-12$ & -4.22 & t_s_at & ANAPC7 & $7.7532 \mathrm{E}-11$ & -4.210797517 \\
\hline 229538_s_at & IQGAP3 & $3.39373 \mathrm{E}-12$ & -4.676 & 204649_at & TROAP & $8.73972 \mathrm{E}-11$ & 9358 \\
\hline 38158_at & ESPL1 & $3.52074 \mathrm{E}-12$ & -4.330276 & 212871_at & MAPKAPK5 & $9.64493 \mathrm{E}-11$ & -6.062517519 \\
\hline 224753_at & CDCA5 & 3.816 & -3. & 201954_at & ARPC1B & $1.04984 \mathrm{E}-10$ & -3.29272791 \\
\hline 200044_at & SRSF9 & $5.19895 \mathrm{E}-12$ & -4 & 203967_at & CDC6 & $1.15562 \mathrm{E}-10$ & -3.032999971 \\
\hline 234915_s_at & DENR & 6.646 & -3 & 205024_s_at & RAD51 & $1.27276 \mathrm{E}-10$ & -3.317013997 \\
\hline 206316_s_at & KNTC1 & E-12 & $\begin{array}{l}-5 \\
-3\end{array}$ & 201127_s_at & ACLY & $1.40898 \mathrm{E}-10$ & -3.598775099 \\
\hline 225468_at & PATL1 & $\begin{array}{l}12 \\
12\end{array}$ & $\begin{array}{l}-3 \\
-4\end{array}$ & 201292_at & TOP2A & $1.69439 \mathrm{E}-10$ & -3.586121076 \\
\hline 200756_x_at & CALU & 12 & -3 & 1555274_a_at & EPT1 & $1.82091 \mathrm{E}-10$ & -3.107139925 \\
\hline 202095_s_at & BIRC5 & 12 & -3 & 222077_s_at & RACGAP1 & $1.98689 \mathrm{E}-10$ & -3.463568797 \\
\hline 209464_at & AUR & 2 & -5 & 212949_at & NCAPH & $2.04934 \mathrm{E}-10$ & -3.123094613 \\
\hline 204430_s_at & SLC & & & 214866_at & PLAUR & $2.8521 \mathrm{E}-10$ & -6.066208054 \\
\hline 219918_s_at & & & & 209836_x_at & BOLA2B & $3.03036 \mathrm{E}-10$ & -3.581736948 \\
\hline 218512 at & & & & 236957_at & CDCA2 & $3.37438 \mathrm{E}-10$ & -3.267349523 \\
\hline 203702 s at & & 1 10745Г 11 & & 204318_s_c & GTSE1 & $3.6192 \mathrm{E}-10$ & -3.165321627 \\
\hline & TTLL4 & $1.10745 \mathrm{E}-11$ & -3.222581427 & 222622_at & PGP & $3.89473 \mathrm{E}-10$ & -3.166188967 \\
\hline 242944_at & FAM83A & $1.14144 \mathrm{E}-11$ & -6.56980268 & 218497 s at & RNASEH1 & $4.25561 \mathrm{E}-10$ & -3.276072648 \\
\hline 206205_at & MPHOSPH9 & $1.17426 \mathrm{E}-11$ & -3.286743793 & 218984_at & PUS7 & $4.45897 \mathrm{E}-10$ & -4.331098443 \\
\hline 221520_s_at & CDCA8 & $1.222 \mathrm{E}-11$ & -3.189226567 & 205394_at & CHEK1 & $4.6472 \mathrm{E}-10$ & -3.071160119 \\
\hline 220011_at & AUNIP & $1.32323 \mathrm{E}-11$ & -5.645650742 & 10821 x & CENPA & $4.95303 \mathrm{E}-10$ & -3.345790152 \\
\hline 203004_s_at & MEF2D & $1.41975 \mathrm{E}-11$ & -6.628593875 & $\begin{array}{l}210821 \text { X_c } \\
223484 \text { at }\end{array}$ & C15orf48 & $6.08452 \mathrm{E}-10$ & -3.301630777 \\
\hline 204005_s_at & PAWR & $1.44695 \mathrm{E}-11$ & -4.589047842 & 13523 at & CCNE1 & $6.55394 \mathrm{E}-10$ & -4.360746545 \\
\hline 200744_s_at & GNB1 & $1.57292 \mathrm{E}-11$ & -3.309783419 & 1J525_at & BUB1 & $7.26076 \mathrm{E}-10$ & -3.325492652 \\
\hline 202580_x_at & FOXM1 & $1.92268 \mathrm{E}-11$ & -3.156340828 & 202240_at & PLK1 & $8.52925 \mathrm{E}-10$ & -3.537560833 \\
\hline 201761_at & MTHFD2 & $2.141 \mathrm{E}-11$ & -3.158744955 & & & & \\
\hline
\end{tabular}

Table II. Continued. 
Table III. The 100 downregulated genes.

\begin{tabular}{|c|c|c|c|c|c|c|c|}
\hline Probe ID & Gene & P-value & Z-score & Probe ID & Gene & P-value & Z-score \\
\hline 225956_at & CREBRF & 0 & 3.056084 & 225811_at & C11orf58 & $2.90212 \mathrm{E}-13$ & 3.095344537 \\
\hline 209740_s_at & PNPLA4 & 0 & 8.750866 & 227847_at & EPM2AIP1 & $3.27738 \mathrm{E}-13$ & 3.460553723 \\
\hline 204754_at & HLF & 0 & 3.370263 & 201019_s_at & EIF1AX & $3.35065 \mathrm{E}-13$ & 4.257274339 \\
\hline 230163_at & GFRA1 & 0 & 3.162875 & 223695_s_at & ARSD & $3.475 \mathrm{E}-13$ & 5.635180257 \\
\hline 242496_at & ART4 & 0 & 3.160279 & 228905_at & PCM1 & $3.53051 \mathrm{E}-13$ & 3.340750721 \\
\hline 221518_s_at & USP47 & 0 & 4.047036 & 217707_x_at & SMARCA2 & $3.67262 \mathrm{E}-13$ & 4.020194349 \\
\hline 235830_at & NT5DC1 & 0 & 3.951365 & 225093_at & UTRN & $6.21503 \mathrm{E}-13$ & 3.138806562 \\
\hline 235155_at & $\mathrm{BDH} 2$ & 0 & 3.138416 & 227425_at & REPS2 & $7.33413 \mathrm{E}-13$ & 3.055352168 \\
\hline 208741_at & SAP18 & 0 & 3.588813 & 211734_s_at & FCER1A & $8.45324 \mathrm{E}-13$ & 3.411503985 \\
\hline 228692_at & PREX2 & 0 & 3.033953 & 244007_at & ZNF462 & $9.36362 \mathrm{E}-13$ & 3.786986943 \\
\hline 211999_at & MIR4738 & 0 & 3.297597 & 212675_s_at & CEP68 & $1.00742 \mathrm{E}-12$ & 3.307657084 \\
\hline 227562_at & LAMTOR3 & 0 & 3.340261 & 238454_at & ZNF540 & $1.13221 \mathrm{E}-12$ & 3.186059238 \\
\hline 229573_at & USP9X & $2.22 \mathrm{E}-16$ & 4.870675 & 224889_at & FOXO3 & $1.14175 \mathrm{E}-12$ & 3.853408162 \\
\hline 205756_s_at & F8 & $2.22 \mathrm{E}-16$ & 3.20333 & 1558512_at & RP11-819C21.1 & $1.37579 \mathrm{E}-12$ & 3.144887286 \\
\hline 229319_at & BC022047 & $2.22 \mathrm{E}-16$ & 3.024973 & 213802_at & PRSS12 & $1.47216 \mathrm{E}-12$ & 4.357472705 \\
\hline 228411_at & PARD3B & $4.44 \mathrm{E}-16$ & 3.454669 & 225465_at & MAGI1 & $1.47393 \mathrm{E}-12$ & 4.208157151 \\
\hline 212425_at & SCAMP1 & $4.44 \mathrm{E}-16$ & 3.064577 & 223126_s_at & C1orf21 & $1.56142 \mathrm{E}-12$ & 3.186640389 \\
\hline 213876_x_at & ZRSR2 & $4.44 \mathrm{E}-16$ & 5.174619 & 230479_at & EIF3F & $1.58984 \mathrm{E}-12$ & 3.299359045 \\
\hline 239252_at & COX7B & $4.44 \mathrm{E}-16$ & 3.999039 & 228448_at & MAP6 & $1.66223 \mathrm{E}-12$ & 3.143593284 \\
\hline 200933_x_at & RPS4X & $4.44 \mathrm{E}-16$ & 5.299386 & 217779_s_at & PNRC2 & $1.91847 \mathrm{E}-12$ & 3.246325539 \\
\hline 210829_s_at & SSBP2 & $4.44 \mathrm{E}-16$ & 3.082665 & 1560648_s_at & TSPYL1 & $1.9309 \mathrm{E}-12$ & 3.760805629 \\
\hline 206767_at & RBMS3 & $6.66 \mathrm{E}-16$ & 3.71459 & 212936_at & FAM172A & $2.19358 \mathrm{E}-12$ & 4.299840018 \\
\hline 226709_at & ROBO2 & $6.66 \mathrm{E}-16$ & 3.615428 & 227091_at & CCDC146 & $2.29194 \mathrm{E}-12$ & 3.206298087 \\
\hline 203991_s_at & KDM6A & $8.88 \mathrm{E}-16$ & 5.796073 & 221564_at & PRMT2 & $2.38565 \mathrm{E}-12$ & 3.547995663 \\
\hline 227274_at & SYNJ2BP-COX16 & $1.11 \mathrm{E}-15$ & 3.517758 & 43427_at & $\mathrm{ACACB}$ & $2.44649 \mathrm{E}-12$ & 3.004593504 \\
\hline 228504_at & SCN7A & $1.78 \mathrm{E}-15$ & 3.16819 & 229384_at & СТC-429P9.3 & $2.57394 \mathrm{E}-12$ & 3.228782722 \\
\hline 225998_at & GAB 1 & $2 \mathrm{E}-15$ & 3.00431 & 222663_at & RIOK2 & $2.69118 \mathrm{E}-12$ & 3.35934368 \\
\hline 218346_s_at & SESN1 & $2.44 \mathrm{E}-15$ & 3.055691 & 238472_at & FBXO9 & $2.69273 \mathrm{E}-12$ & 3.562133246 \\
\hline 224976_at & NFIA & $3.11 \mathrm{E}-15$ & 3.007387 & 222533_at & CRBN & $2.82396 \mathrm{E}-12$ & 3.004216036 \\
\hline 205857_at & SLC18A2 & $4.22 \mathrm{E}-15$ & 3.457499 & 228751_at & CLK4 & $3.30425 \mathrm{E}-12$ & 3.359190366 \\
\hline 225352_at & SEC62 & $6.88 \mathrm{E}-15$ & 3.26132 & 208832_at & ATXN10 & $3.36042 \mathrm{E}-12$ & 3.408974266 \\
\hline 200810_s_at & CIRBP & $1.49 \mathrm{E}-14$ & 3.072028 & 238043_at & ARID1B & $3.38618 \mathrm{E}-12$ & 3.280003422 \\
\hline 200983_x_at & CD59 & $2.22 \mathrm{E}-14$ & 3.24769 & 1559412_at & LINC00478 & $3.50475 \mathrm{E}-12$ & 4.041998876 \\
\hline 212249_at & PIK3R1 & $2.44 \mathrm{E}-14$ & 4.98666 & 238081_at & WDFY3-AS2 & $3.68106 \mathrm{E}-12$ & 3.077236586 \\
\hline 241689_at & METTL14 & $3.42 \mathrm{E}-14$ & 3.311901 & 228760_at & SRSF8 & 4.13358E-12 & 3.538832842 \\
\hline 228716_at & THRB & $4.88 \mathrm{E}-14$ & 3.021776 & 235240_at & ATXN3 & 4.47198E-12 & 3.59474854 \\
\hline 205259_at & NR3C2 & $5 \mathrm{E}-14$ & 3.392261 & 240806_at & RPL15 & $5.22404 \mathrm{E}-12$ & 3.229351616 \\
\hline 223588_at & THAP2 & $5.44 \mathrm{E}-14$ & 6.445672 & 228027_at & GPRASP2 & 5.30198E-12 & 3.191435286 \\
\hline 201427_s_at & SEPP1 & $6.02 \mathrm{E}-14$ & 3.146142 & 209815_at & PTCH1 & $5.63194 \mathrm{E}-12$ & 3.080285017 \\
\hline 219427_at & FAT4 & $7.7 \mathrm{E}-14$ & 3.056389 & 208760_at & UBE2I & $6.31295 \mathrm{E}-12$ & 3.075043093 \\
\hline 209807_s_at & NFIX & $7.97 \mathrm{E}-14$ & 3.105386 & 229317_at & KPNA5 & $6.53722 \mathrm{E}-12$ & 3.749106743 \\
\hline 201498_at & USP7 & $8.55 \mathrm{E}-14$ & 3.827248 & 228420_at & PDCD2 & $7.1736 \mathrm{E}-12$ & 3.442288871 \\
\hline 228243_at & RP11-5C23.1 & $8.84 \mathrm{E}-14$ & 3.43588 & 227520_at & TXLNG & 7.54685E-12 & 5.386988658 \\
\hline 238786_at & ANK3 & $1.58 \mathrm{E}-13$ & 3.075604 & 244294_at & GTF2H5 & 7.70273E-12 & 4.035395557 \\
\hline 233249_at & LOC100507073 & $1.61 \mathrm{E}-13$ & 3.069721 & 204011_at & SPRY2 & $7.75358 \mathrm{E}-12$ & 3.811245705 \\
\hline 208633_s_at & MACF1 & $1.79 \mathrm{E}-13$ & 3.260397 & 209614_at & ADH1B & $7.83396 \mathrm{E}-12$ & 3.188622844 \\
\hline 226816_s_at & KIAA1143 & $1.94 \mathrm{E}-13$ & 3.431996 & 226774_at & FAM120B & $8.43059 \mathrm{E}-12$ & 3.286960689 \\
\hline 208792_s_at & CLU & $2.46 \mathrm{E}-13$ & 3.627978 & 235612_at & PRPF38A & $1.023 \mathrm{E}-11$ & 3.636955078 \\
\hline 210426_x_at & RORA & $2.51 \mathrm{E}-13$ & 3.077789 & 232122_s_at & VEPH1 & $1.20886 \mathrm{E}-11$ & 3.052642894 \\
\hline 229969_at & SEC63 & $2.86 \mathrm{E}-13$ & 3.019815 & 216342_x_at & RPS4XP2 & $1.22578 \mathrm{E}-11$ & 6.967247025 \\
\hline
\end{tabular}

Table III. Continued. 


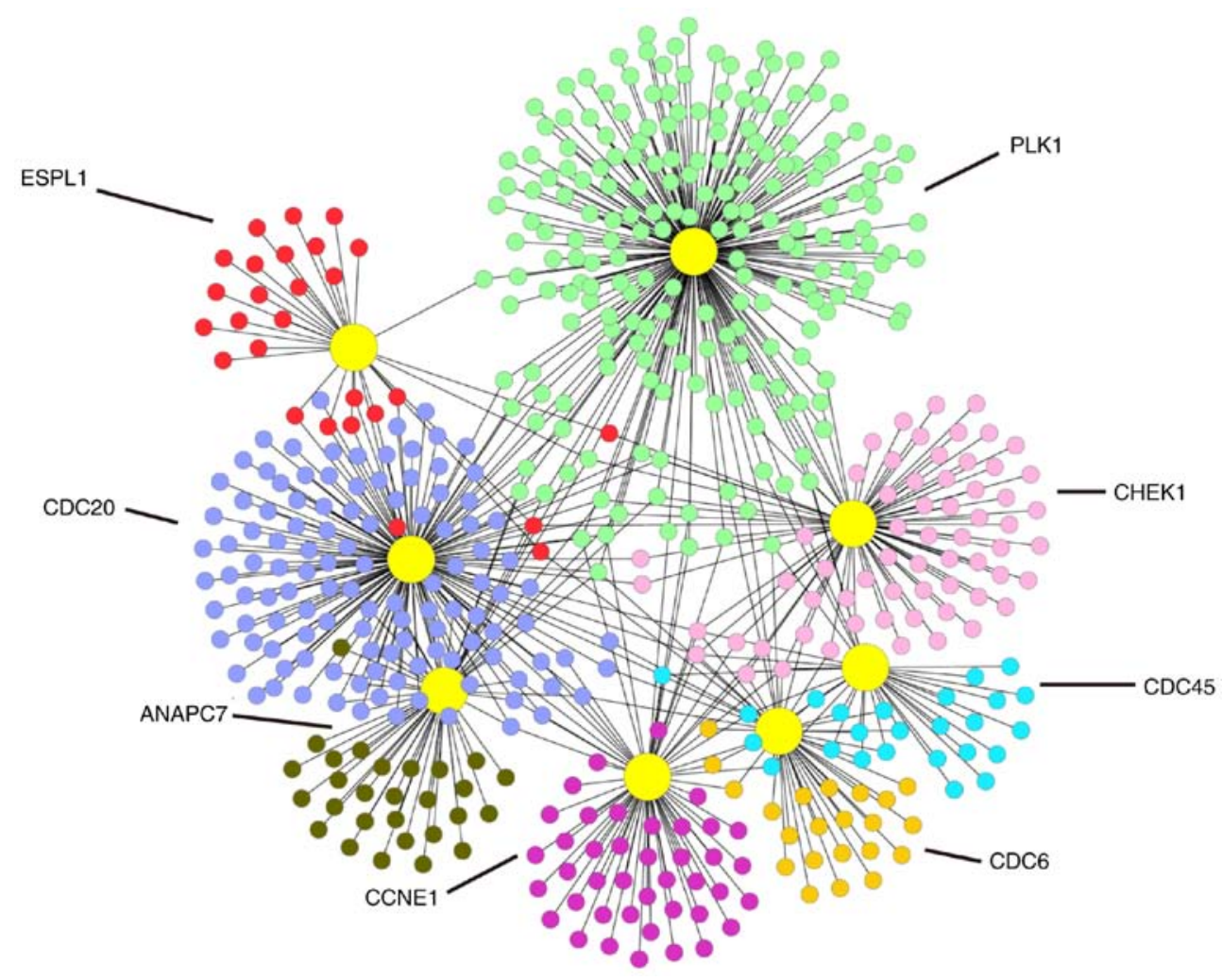

Figure 7. Protein-protein interaction (PPI) network of the 8 upregulated differentially expressed genes (DEGs).

Table IV. The enrichment based on the top $10 \mathrm{GO}$ functions shows the top 100 upregulated and downregulated DEGs.

\begin{tabular}{llrl}
\hline GO ID & \multicolumn{1}{c}{ GO term } & $\begin{array}{r}\text { No. of } \\
\text { Genes }\end{array}$ & P-value \\
\hline GO:0022403 & Cell cycle phase & 48 & $3.26 \mathrm{E}-18$ \\
GO:0000087 & $\begin{array}{l}\text { M phase of mitotic } \\
\text { cell cycle }\end{array}$ & 33 & $6.78 \mathrm{E}-18$ \\
GO:0022402 & Cell cycle process & 52 & $6.78 \mathrm{E}-18$ \\
GO:0000278 & Mitotic cell cycle & 45 & $6.78 \mathrm{E}-18$ \\
GO:0044428 & Nuclear part & 70 & $6.12 \mathrm{E}-10$ \\
GO:0031981 & Nuclear lumen & 64 & $1.48 \mathrm{E}-09$ \\
GO:0044422 & Organelle part & 112 & $1.63 \mathrm{E}-09$ \\
GO:0005515 & Protein binding & 112 & $1.27 \mathrm{E}-05$ \\
GO:0042975 & Peroxisome proliferator & 3 & 0.0097 \\
& activated receptor binding & & \\
GO:0019899 & Enzyme binding & 25 & 0.0135 \\
\hline
\end{tabular}

GO, gene ontology; DEGs, differentially expressed genes.

Using several perspectives would allow us to characterise the underlying mechanisms of lung adenocarcinoma in smokers. Thus, we performed a meta-analysis of four independent microarray datasets using the same platform. The large number of DEGs identified in our study implies that our approach produces more reliable results in identifying differences in gene expression levels among lung adenocarcinoma patients who either had a smoking or a non-smoking history.
Table V. The enrichment based on the top KEGG pathway shows the top 100 upregulated and downregulated DEGs.

\begin{tabular}{llcl}
\hline KEGG ID & \multicolumn{1}{c}{ KEGG pathway } & $\begin{array}{c}\text { No. of } \\
\text { Genes }\end{array}$ & P-value \\
\hline kegg:04110 & Cell cycle & 8 & $2.45 \mathrm{E}-06$ \\
kegg:04114 & Oocyte meiosis & 7 & $9.76 \mathrm{E}-06$ \\
kegg:04120 & Ubiquitin mediated & 5 & 0.0032 \\
& proteolysis & & \\
kegg:03013 & RNA transport & 5 & 0.0036 \\
kegg:04610 & Complement and & 3 & 0.013 \\
& coagulation cascades & & \\
kegg:04115 & p53 signalling pathway & 3 & 0.013 \\
kegg:05200 & Pathways in cancer & 6 & 0.013 \\
kegg:03060 & Protein export & 2 & 0.0144 \\
kegg:03008 & Ribosome biogenesis in & 3 & 0.0152 \\
& eukaryotes & & \multirow{2}{*}{ kegg:03440 } \\
Homologous recombination & 2 & 0.0168 \\
\hline
\end{tabular}

KEGG, Kyoto Encyclopedia of Genes and Genomes; DEGs, differentially expressed genes.

In this study, the microarray expression datasets derived from lung adenocarcinoma tissue with patients with either a smoking or non-smoking history were publicly available. A number of previous studies have molecularly characterised the genetic profiles in lung cancer patients with or without a smoking history. The present investigation focused on a relatively larger 
cohort with 477 lung adenocarcinoma tissues from 327 smoking patients and 150 non-smoking patients, thereby providing a more powerful analysis. Our study results were highly consistent with previous DEG analyses, supporting the utility and validity of this analytical approach. Additionally, it also revealed that multiple biological processes and pathways, including cell cycle phase and the cell cycle pathway, were significantly affected in lung adenocarcinoma tissues from smoking patients compared to the non-smoking patients. Consistently, many previous studies have revealed that cigarette smoke extract accelerated premature gene mutations in the cell cycle pathway. Cigarette smoke extract alters the cell cycle via the phospholipid transfer protein/transforming growth factor- $\beta 1 /$ cyclinD1/CDK4 pathway (24). Cigarette smoking is a major factor for many cancers including, pancreatic cancer, human ovarian cancer and colon cancer (25-27). This study identified the 8 overexpressed genes in the cell cycle pathway as CDC45, PLK1, CDC20, ANAPC7, CDC6, CHEK1, CCNE1 and ESPL1. According to the P-values in the meta-analysis, we identified a few significant DEGs including CDC45, CDC20, ANAPC7, CDC6, and ESPL1. Based on our meta-analysis results, these five genes may be potential target genes for the treatment of this disease.

CDC45 is a member of the highly conserved multiprotein complex including $\mathrm{Cdc} 6 / \mathrm{Cdc} 18$. The replication factor CDC45 has essential functions in the initiation and plays an important role in the intra-S-phase checkpoint (28). CDC45 has been found to be upregulated in many neoplasms, such as breast neoplasms, colorectal neoplasms, lung neoplasms and haematological neoplasms (29).

CDC20 appears to act as a regulatory protein by interacting with several other proteins at multiple points in the cell cycle (30). The CDC20 gene might play an important role in the malignancy of NSCLC. Additionally, CDC20 has been found to be upregulated in lung cancer patients with a smoking history (31). In addition, through this analysis, we identified the overexpression of the CDC20 gene in lung adenocarcinoma patients who had a smoking history compared to the non-smoking patients. Combined with previous research, our analysis demonstrates that the CDC20 gene might play an important role in the treatment of lung adenocarcinoma in smoking patients.

ANAPC7 is an E3 ligase enzyme that ubiquinates various proteins involved in the cell cycle (32). This protein complex may have a pivotal role in the cell cycle control affecting pathological conditions such as cancer (33). ANAPC mutations have been reported in lung squamous cell carcinoma and small cell lung carcinoma.

CDC6, a cell cycle regulatory gene, is an essential regulator of DNA replication and plays important roles in the activation and maintenance of the checkpoint mechanism in the cell cycle (34). CDC6 has been associated with the oncogenic activities in human cancers, such as ovarian cancer, lung cancer and prostate cancer $(35,36)$. However, the biological function and clinical significance of CDC6 in lung adenocarcinoma remain unclear. A previous study suggests that CDC6 is associated with the decline in lung function of ex-smoking in COPD (37). Our study also revealed CDC6 overexpression in lung adenocarcinoma patients with a smoking history compared to non-smoking patients.

ESPL1 is a protein-coding gene, and its overexpression has been found in a variety of human cancers such as rectum adenocarcinoma, prostate carcinoma, breast carcinoma and lung carcinoma $(38,39)$. Consistent with earlier results, our study revealed that ESPL is overexpressed in lung adenocarcinoma in patients with a smoking history compared to those who had a non-smoking history.

Overall, the present study identified that a few genes are differentially expressed in lung adenocarcinoma samples between smoker and non-smoker patients. This observation supports previous studies; however, our analysis provides new insights that enable better understanding of the molecular mechanisms of lung adenocarcinoma in smokers, which may provide potential targets for the therapeutic design of individualized treatments for lung adenocarcinoma patients who have a smoking history.

\section{Acknowledgements}

This research was supported in part by grants form the National Natural Science Foundation of China (31560314 to Q.L.) and the Natural Science Foundation of Jiangxi Province (2016BAB204168 to Q.L.).

\section{References}

1. Kalemkerian GP, Akerley W, Bogner P, Borghaei H, Chow LQ, Downey RJ, Gandhi L, Ganti AK, Govindan R, Grecula JC, et al; National Comprehensive Cancer Network: Small cell lung cancer. J Natl Compr Canc Netw 11: 78-98, 2013.

2. Pasche B and Grant SC: Non-small cell lung cancer and precision medicine: A model for the incorporation of genomic features into clinical trial design. JAMA 311: 1975-1976, 2014.

3. Saito M, Shiraishi K, Kunitoh H, Takenoshita S, Yokota J and Kohno T: Gene aberrations for precision medicine against lung adenocarcinoma. Cancer Sci 107: 713-720, 2016.

4. Ding L, Getz G, Wheeler DA, Mardis ER, McLellan MD Cibulskis K, Sougnez C, Greulich H, Muzny DM, Morgan MB, et al: Somatic mutations affect key pathways in lung adenocarcinoma. Nature 455: 1069-1075, 2008.

5. Weir BA, Woo MS, Getz G, Perner S, Ding L, Beroukhim R, Lin WM, Province MA, Kraja A, Johnson LA, et al: Characterizing the cancer genome in lung adenocarcinoma. Nature 450: 893-898, 2007.

6. de Mello RA, Madureira P, Carvalho LS, Araújo A, O'Brien M and Popat S: EGFR and KRAS mutations, and ALK fusions: Current developments and personalized therapies for patients with advanced non-small-cell lung cancer. Pharmacogenomics 14: 1765-1777, 2013.

7. Paul D and Rafael OC: Concurrent targetable genetic driver alterations in KRAS-mutant lung adenocarcinoma. Eur J Cancer 60: e15-e16, 2016.

8. Nie Q, Yang XN, An SJ, Zhang XC, Yang JJ, Zhong WZ, Liao RQ, Chen ZH, Su J, Xie Z, et al: CYP1A1*2A polymorphism as a predictor of clinical outcome in advanced lung cancer patients treated with EGFR-TKI and its combined effects with EGFR intron 1 (CA)n polymorphism. Eur J Cancer 47: 1962-1970, 2011

9. Gow CH, Chang HT, Lim CK, Liu CY, Chen JS and Shih JY: Comparable clinical outcomes in patients with HER2-mutant and EGFR-mutant lung adenocarcinomas. Genes Chromosomes Cancer 56: 373-381, 2017.

10. Raponi M, Winkler H and Dracopoli NC: KRAS mutations predict response to EGFR inhibitors. Curr Opin Pharmacol 8: 413-418, 2008.

11. Wu C, Zhu J and Zhang X: Network-based differential gene expression analysis suggests cell cycle related genes regulated by E2F1 underlie the molecular difference between smoker and non-smoker lung adenocarcinoma. BMC Bioinformatics 14: 365, 2013.

12. Andreev K, Trufa ID, Siegemund R, Rieker R, Hartmann A, Schmidt J, Sirbu H and Finotto S: Impaired T-bet-pSTAT1 $\alpha$ and perforin-mediated immune responses in the tumoral region of lung adenocarcinoma. Br J Cancer 113: 902-913, 2015. 
13. Talhout R, Schulz T, Florek E, van Benthem J, Wester P and Opperhuizen A: Hazardous compounds in tobacco smoke. Int J Environ Res Public Health 8: 613-628, 2011.

14. Li Y, Xiao X, Ji X, Liu B and Amos CI: RNA-seq analysis of lung adenocarcinomas reveals different gene expression profiles between smoking and nonsmoking patients. Tumour Biol 36: 8993-9003, 2015.

15. Chee M, Yang R, Hubbell E, Berno A, Huang XC, Stern D, Winkler J, Lockhart DJ, Morris MS and Fodor SP: Accessing genetic information with high-density DNA arrays. Science 274 610-614, 1996.

16. Spies M, Dasu MR, Svrakic N, Nesic O, Barrow RE, Perez-Polo JR and Herndon DN: Gene expression analysis in burn wounds of rats. Am J Physiol Regul Integr Comp Physiol 283: R918-R930, 2002.

17. Guo QM: DNA microarray and cancer. Curr Opin Oncol 15: $36-43,2003$

18. Li R, Wang H, Bekele BN, Yin Z, Caraway NP, Katz RL, Stass SA and Jiang F: Identification of putative oncogenes in lung adenocarcinoma by a comprehensive functional genomic approach. Oncogene 25: 2628-2635, 2006.

19. Yasrebi H: Comparative study of joint analysis of microarray gene expression data in survival prediction and risk assessment of breast cancer patients. Brief Bioinform 17: 771-785, 2016.

20. Hong F, Breitling R, McEntee CW, Wittner BS, Nemhauser JL and Chory J: RankProd: A bioconductor package for detecting differentially expressed genes in meta-analysis. Bioinformatics 22: 2825-2827, 2006

21. Hecht SS: Tobacco carcinogens, their biomarkers and tobaccoinduced cancer. Nat Rev Cancer 3: 733-744, 2003.

22. Brüske-Hohlfeld I: Environmental and occupational risk factors for lung cancer. Methods Mol Biol 472: 3-23, 2009.

23. Taioli E: Gene-environment interaction in tobacco-related cancers. Carcinogenesis 29: 1467-1474, 2008.

24. Chai XM, Li YL, Chen H, Guo SL, Shui LL and Chen YJ: Cigarette smoke extract alters the cell cycle via the phospholipid transfer protein/transforming growth factor- $\beta 1 /$ CyclinD1/CDK4 pathway. Eur J Pharmacol 786: 85-93, 2016.

25. Glauert HP, Elliott RS, Han SG, Athey M, Lee EY and Gairola CG: Effect of cigarette smoke exposure and mutant Kras overexpression on pancreatic cell proliferation. Oncol Lett 13: 1939-1943, 2017.

26. Jeon SY, Go RE, Heo JR, Kim CW, Hwang KA and Choi KC: Effects of cigarette smoke extracts on the progression and metastasis of human ovarian cancer cells via regulating epithelialmesenchymal transition. Reprod Toxicol 65: 1-10, 2016.

27. Schmidt U, Wollmann Y, Franke C, Grosse F, Saluz HP and Hänel F: Characterization of the interaction between the human DNA topoisomerase IIbeta-binding protein 1 (TopBP1) and the cell division cycle 45 (Cdc45) protein. Biochem J 409: 169-177, 2008 .
28. Tercero JA, Longhese MP and Diffley JFX: A central role for DNA replication forks in checkpoint activation and response. Mol Cell 11: 1323-1336, 2003.

29. Broderick R, Rainey MD, Santocanale C and Nasheuer HP: Cell cycle-dependent formation of Cdc45-Claspin complexes in human cells is compromized by UV-mediated DNA damage. FEBS J 280: 4888-4902, 2013.

30. Weinstein J: Cell cycle-regulated expression, phosphorylation, and degradation of p55Cdc. A mammalian homolog of CDC20/ Fizzy/slp1. J Biol Chem 272: 28501-28511, 1997.

31. Pollok S, Bauerschmidt C, Sänger J, Nasheuer HP and Grosse F Human Cdc45 is a proliferation-associated antigen. FEBS J 274: 3669-3684, 2007.

32. Rahimi H, Ahmadzadeh A, Yousef-amoli S, Kokabee L, Shokrgozar MA, Mahdian R and Karimipoor M: The expression pattern of APC2 and APC7 in various cancer cell lines and AML patients. Adv Med Sci 60: 259-263, 2015.

33. Zhang X, Xiao T, Cheng S, Tong T and Gao Y: Cigarette smoke suppresses the ubiquitin-dependent degradation of OLC1. Biochem Biophys Res Commun 407: 753-757, 2011.

34. Borlado LR and Méndez J: CDC6: From DNA replication to cell cycle checkpoints and oncogenesis. Carcinogenesis 29: 237-243, 2008.

35. Sun T-Y, Xie H-J, Li Z, He H and Kong L-F: Expression of CDC6 in ovarian cancer and its effect on proliferation of ovarian cancer cells. Int J Clin Exp Med 9: 10544-10550, 2016.

36. Yun SJ, Kim YH, Kang HW, Kim WT, Kim YJ, Lee SC, Kim W-J and Kim T: CDC6 mRNA expression is associated with the aggressiveness of prostate cancer. Eur Urol Suppl 15: e1625, 2016.

37. Takabatake N, Toriyama S, Igarashi A, Tokairin Y, Takeishi Y, Konta T, Inoue S, Abe S, Shibata Y and Kubota I: A novel polymorphism in CDC6 is associated with the decline in lung function of ex-smokers in COPD. Biochem Biophys Res Commun 381: 554-559, 2009.

38. Finetti P, Guille A, Adelaide J, Birnbaum D, Chaffanet M and Bertucci F: ESPL1 is a candidate oncogene of luminal B breast cancers. Breast Cancer Res Treat 147: 51-59, 2014.

39. Zhang $\mathrm{N}$ and Pati D: Biology and insights into the role of cohesin protease separase in human malignancies. Biol Rev Camb Philos Soc 92: 2070-2083, 2017.

This work is licensed under a Creative Commons Attribution-NonCommercial-NoDerivatives 4.0 International (CC BY-NC-ND 4.0) License. 